\title{
An Unusual Small Bowel Mass: India Ink Tattooing Mimicking Malignancy
}

\author{
Kunal Kochar, MD, Ajit Pai, MD, Slawomir Marecik, MD, John Park, MD, Leela Prasad, MD \\ Division of Colon and Rectal Surgery, Advocate Lutheran General Hospital, Park Ridge, IL, USA (all authors).
}

\begin{abstract}
Introduction: India ink is routinely used for preoperative marking of colonic lesions to facilitate identification during laparoscopic colon surgery. It is a relatively inert dye with few reported adverse effects.

Case Description: We report a case of inadvertent extracolonic tattooing and intra-peritoneal spillage of India ink leading to adhesions and formation of a mass, which mimicked malignancy. The relevant literature is also reviewed.

Discussion: Although India ink is a safe dye for colonic tattooing and most of its complications are asymptomatic, it can occasionally lead to complications that might mimic malignancy and cause a diagnostic dilemma.

Key Words: India ink, Small bowel mass, Malignancy, Complication, Endoscopy.

Citation Kochar K, Pai A, Maricek S, Park J, Prasad L. An unusual small bowel mass: India ink tattooing mimicking malignancy. CRSLS e2014.00090. DOI: 10.4293/CRSLS.2014.00090.

Copyright $\odot 2014$ SLS This is an open-access article distributed under the terms of the Creative Commons Attribution-Noncommercial-ShareAlike 3.0 Unported license, which permits unrestricted noncommercial use, distribution, and reproduction in any medium, provided the original author and source are credited.

Address correspondence to: Kunal Kochar, MD, Division of Colon and Rectal Surgery, Advocate Lutheran General Hospital, 1150 N Northwest Hwy, Ste 107, Park Ridge, IL 60068, USA. Telephone: (847) 759-1110. E-mail: kocharkunall@gmail.com
\end{abstract}

\section{INTRODUCTION}

The laparoscopic approach to colorectal cancer is now widely accepted. With the reduction of tactile feedback in laparoscopic surgery, preoperative visual marking of the lesion is essential. Of the various modalities for preoperative localization, colonoscopic tattooing with India ink is the most widely used method. It is safe, with a low incidence of complications $(0.22 \%),{ }^{1}$ and remains for a prolonged duration.

We report a case of asymptomatic small bowel abscess due to India ink injection, which mimicked malignancy.

\section{CASE REPORT}

A 49-year-old man with no significant medical history presented to his gastroenterologist with a 1-month history of rectal bleeding and new-onset incontinence for liquid feces. He underwent a colonoscopy, which showed a rectosigmoid tumor. The tumor underwent biopsy and was tattooed with India ink. The rest of the colonoscopy yielded normal findings. A total of $4 \mathrm{~mL}$ of India ink (Spot; GI Supply, Camp Hill, Pennsylvania) was used to tattoo the tumor circumferentially using a 23-gauge sclerotherapy needle. Pathologic examination of the colonoscopic biopsy specimens showed a moderately differentiated adenocarcinoma. During laparoscopic low anterior resec- tion, extensive adhesions were seen and a loop of small bowel was found to be adherent to the sigmoid colon, with black pigment staining seen on the surface of the bowel (Figure 1). A Pfannenstiel incision was made, and a hand port was inserted to further assist in assessment. Clinically, it was difficult to differentiate an inflammatory process involving the small bowel from a primary or metastatic malignant process. The small bowel loop was therefore resected en bloc with the sigmoid colon containing the tumor. On gross pathologic assessment, the serosa of the small bowel showed an area of black tattoo ink and dense fibrosis with adherent mesenteric fat. Microscopically, the segment of small bowel showed transmural abscess with fibrosis, fat necrosis, and black tattoo pigment. There was no evidence of malignancy at the resection margins. The sigmoid colon showed a moderately differentiated adenocarcinoma invading through the colon wall into the pericolonic fat. Of 23 resected lymph nodes, 1 contained metastasis (pT3pN1aM0).

\section{DISCUSSION}

A variety of endoscopic techniques have been developed to assist in intraoperative localization of colonic lesions. These include tattooing the intestine with substances such as methylene blue, indigo carmine, indocyanine green, or India ink; placement of mucosal clips ${ }^{2,3}$; and intraoperative endoscopy. Dyes such as methylene blue disappear 


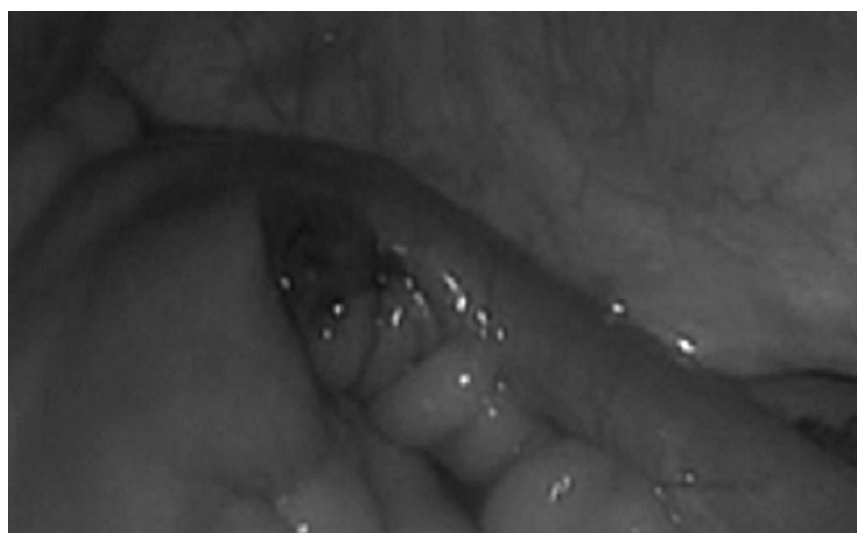

Figure 1. Small bowel adherent to colon with India ink stain.

within a few days because they are rapidly absorbed. India ink, however, is superior because of its lack of diffusion through the mesentery and the inability of the local lymphatics to remove the inert particles. ${ }^{4}$

Adverse effects of India ink injection are rare. Reported asymptomatic complications include fat necrosis with inflammatory pseudotumor formation, ${ }^{5}$ colonic abscess, ${ }^{6}$ and phlegmonous gastritis. ${ }^{7}$ Symptomatic complications include a case of idiopathic inflammatory bowel disease ${ }^{8}$ and localized peritonitis due to accidental marking of the small bowel. 9 The exact mechanism by which India ink results in an adverse reaction is unclear. Carbon itself is inert and is localized mainly in the macrophages of the lamina propria or submucosa. An adverse reaction could result from invasion of the gastrointestinal bacteria via the injection site $^{7}$ or from the toxic ingredients in the preparation itself. India ink is a colloidal suspension of carbon particles, with some brands containing substances such as ethylene glycol, sodium tetraborate decahydrate, ammonia, and gelatin. A prospective study evaluating the long-term safety of India ink tattoos did not find any evidence of major clinical or infective complications. ${ }^{10}$ Why it causes an intense inflammatory reaction in some patients is unknown.

Our patient had colonoscopic tattooing performed for his rectosigmoid tumor. It is likely that there was inadvertent perforation of the colon with injection of the small bowel or a transmural injection of India ink with intraperitoneal spillage, which elicited an intense inflammatory response. Our patient had not undergone any previous abdominal surgery, and the extensive adhesions found during surgery were likely due to the inflammatory response to the India ink. In previously reported cases of pseudotumor formation ${ }^{5}$ and colonic abscess, ${ }^{6}$ the inflammatory and fibroblastic responses to India ink were seen on histologic analysis of resected specimens. In our case, besides extensive adhesions, a small bowel loop was thickened and adherent to the colon, and it appeared on intraoperative palpation to be involved by the rectosigmoid malignancy, forming a mass. On histopathologic examination, there was no evidence of malignancy but a transmural foreign-body granuloma with fibrosis incorporating the area of the tattoo was found.

\section{CONCLUSION}

Laparoscopic colon surgery is being performed more often, and use of endoscopic tattooing is likely to increase. Although most India ink injections will not lead to a complication or may remain asymptomatic, our case demonstrates that the inflammatory and fibroblastic response may mimic an intra-abdominal malignancy or confuse the staging of a colon tumor.

\section{References:}

1. Nizam R, Siddiqi N, Landas SK, Kaplan DS, Holtzapple PG. Colonic tattooing with India ink: benefits, risks, and alternatives. Am J Gastroenterol. 1996;91(9):1804-1808.

2. Hammond DC, Lane FR, Mackeigan JM, Passinault WJ. Endoscopic tattooing of the colon: clinical experience. Am Surg. 1993;59(3):205-210.

3. Lane KL, Vallera R, Washington K, Gottfried MR. Endoscopic tattoo agents in the colon. Tissue responses and clinical implications. Am J Surg Pathol. 1996;20(10):1266-1270.

4. Fu KI, Fujii T, Kato S, et al. A new endoscopic tattooing technique for identifying the location of colonic lesions during laparoscopic surgery: a comparison with the conventional technique. Endoscopy. 2001;33(8):687-691.

5. Coman E, Brandt LJ, Brenner S, Frank M, Sablay B, Bennett. Fat necrosis and inflammatory pseudotumor due to endoscopic tattooing of the colon with India ink. Gastrointest Endosc. 1991;37(1):65-68.

6. Park SI, Genta RS, Romeo DP, Weesner RE. Colonic abscess and focal peritonitis secondary to India ink tattooing of the colon. Gastrointest Endosc. 1991;37(1):68-71.

7. Hornig D, Kühn H, Stadelmann O, Bötticher R. Phlegmonous gastritis after Indian ink marking. Endoscopy. 1983;15(4):266-269.

8. Gopal DV, Morava-Protzner I, Miller HA, Hemphill DJ. Idiopathic inflammatory bowel disease associated with colonic tattooing with India ink preparation-case report and review of literature. Gastrointest Endosc. 1999;49(5):636-639.

9. Singh S, Arif A, Fox C, Basnyat P. Complication after preoperative India ink tattooing in a colonic lesion. Dig Surg. 2006;23(5-6):303.

10. Shatz BA, Weinstock LB, Swanson PE, et al. Long-term safety of India ink tattoos in the colon. Gastrointest Endosc. 1997;45:153-156. 